\title{
Increasing entropy reduces perceived numerosity throughout the lifespan
}

\author{
Chuyan $\mathrm{Qu}^{1}$ Nicholas K. DeWind ${ }^{1}$ \& Elizabeth M. Brannon ${ }^{1}$ \\ ${ }^{1}$ University of Pennsylvania, Department of Psychology
}

Correspondence concerning this article should be addressed to Chuyan Qu, Department of Psychology, University of Pennsylvania, 3720 Walnut St, Philadelphia, PA 19104.

Email: qchuyan@sas.upenn.edu

Declarations of Interest: none.

The data described in this manuscript can be located at $\underline{\text { https://osf.io/rz4yk/ }}$ 


\title{
Increasing entropy reduces perceived numerosity throughout the lifespan
}

\begin{abstract}
Illusions that arise from systematic bias in perceiving numerosity serve as a powerful window into the mechanisms supporting our visual number sense. Recent research has shown that similarly oriented elements appear more numerous than randomly oriented elements in an array. However, this coherence illusion is not predicted by dominant models of numerosity perception. Here we examine whether the orientation coherence illusion is a more general byproduct of the effect of entropy on numerical information-processing. Participants engaged in an ordinal numerical comparison task where the color entropy of arrays was manipulated. We found that homogenously colored arrays were perceived as more numerous than entropic colored arrays (Experiments 1 and 2), suggesting that the coherence illusion on numerosity perception is not specific to a particular visual property (e.g., orientation) but instead that the entropy of visual arrays more generally affects numerical processing. In Experiment 3, we explored the developmental trajectory of the color entropy effect in children aged 5 to 17 and found that the strength of the coherence illusion increases into adulthood, raising intriguing questions as to how perceptual experiences influence the progression of this numerosity illusion. We consider a recently proposed resource-rational model as a framework for understanding the entropy effect on numerosity perception under an information-theoretic perspective.
\end{abstract}

Keywords: visual illusions, number sense, numerosity perception, coherence illusion, entropy, resource-rational model 


\section{Introduction}

Number is a fundamental attribute of the world that is readily extracted by the visual system. Humans approximately estimate and compare large sets of items without relying on linguistic symbols or verbal counting (Dehaene, 1997; Feigenson, Dehaene, \& Spelke, 2004). This basic sense of number, formally known as the approximate number system (ANS), emerges early in human development and is shared with a wide range of animal species (Brannon \& Terrace, 1998; Cantlon \& Brannon, 2006; Scarf, Hayne, \& Colombo, 2011; Xu, Spelke, \& Goddard, 2005). A behavioral hallmark of the ANS is Weber's Law: the discriminability of two numerosities is proportional to the ratio between them, but not their absolute difference (e.g., Cantlon, Cordes, Libertus, \& Brannon, 2009). To date, despite considerable investigation, the cognitive mechanisms by which approximate number representations are derived from visual input are largely unknown.

Although number is an abstract property of a visual scene, a wealth of evidence indicates that numerosity judgements are sensitive to a variety of non-numerical features of visual arrays, such as object size, perimeter, density, and spatial frequency (Dakin, Tibber, Greenwood, \& Morgan, 2011; Leibovich, Katzin, Harel, \& Henik, 2017; M. J. Morgan, Raphael, Tibber, \& Dakin, 2014; Starr, DeWind, \& Brannon, 2017). These findings have led to a class of indirect models of numerosity perception which contend that number representations are constructed from a combination of "surrogate" non-numerical magnitudes. For example, according to an early “occupancy model", numerosity judgements are determined by the occupancy index, which is the subjective area of the stimulus field collectively occupied by all items (Allik \& Tuulmets, 1991). Specifically, each item occupies a circular territory of a fixed radius; if the individual territories of two items overlap due to close distance between each other, the occupancy index decreases and consequently leads to underestimation of numerosity. This model well accounts for the spacing 
illusion whereby smaller distance between array items decreases the perceived number of items in the array (e.g., DeWind, Adams, Platt, \& Brannon, 2015; Gebuis \& Reynvoet, 2012). Similarly, this model could explain why a regularly arranged array appears more numerous than a randomly arranged array, known as the "regular-random illusion" because random arrangement is associated with smaller element spacing and larger overlap of the occupied territories of neighboring elements (Ginsburg, 1976). Another more recent model posits that numerosity and spatial frequency perception are inextricably linked (Dakin et al., 2011; M. J. Morgan et al., 2014).

In contrast to these indirect models, an alternative proposal is that numerosity is sensed directly by segmenting entities into discrete units and then summing those units, independent of low-level visual cues. For example, the Dehaene and Changeux (1993) information processing model posits that numerosity is detected through an operation of "object normalization." Objects are initially represented on a simplified input "retina" as blobs of activation. The normalizing operation then segments objects on a topographical map and generates a single active cluster for each, independent of confounds such as size and position. Perhaps the most convincing demonstration in favor of a segmentation account is that binding objects into pairs connected by thin lines substantially reduces their perceived numerosity, whereas introducing a small break between the lines eliminates this effect (Franconeri, Bemis, \& Alvarez, 2009; He, Zhang, Zhou, \& Chen, 2009). More recent studies showed similar connectedness effect using Kanizsa-like illusory contours to simulate physical connections between objects (Adriano, Girelli, \& Rinaldi, 2021; Adriano, Rinaldi, \& Girelli, 2021). Although manipulating connectedness has little effect on nonnumerical cues such as total surface area, density, or spatial frequency, it does change object segmentation and consequently the subjective perception of numerosity. 
None of the above described models predict the recently reported coherence illusion (DeWind, Bonner, \& Brannon, 2020). In a series of experiments, DeWind et al. manipulated the orientation variance (coherence) of arrays containing Gabor patches, such that in the most coherent arrays all the patches pointed to the same direction whereas in the high variance arrays each patch pointed in a different direction. Across four experiments and two different tasks, they found that array-coherence systematically altered subjective numerosity such that more coherent arrays were perceived as more numerous than arrays with variable orientations.

An important question is whether this coherence effect is specific to orientation or is more general. Orientation variance is represented directly by the visual system at an early cortical stage and may play an essential role in perceiving texture and segmenting visual scenes (Dakin, 1999; M. Morgan, Chubb, \& Solomon, 2008; Norman, Heywood, \& Kentridge, 2015). It is therefore possible that orientation variance uniquely interacts with numerosity perception, and is mediated through region-based perception (e.g., texture perception). Although the spacing and element size were consistent for each array in the DeWind et al study, participants may have perceived the more coherently oriented arrays as having greater spacing, and then associated greater perceived spacing with larger numerosity, in a similar way as the "regular-random illusion".

Here we test an alternative possibility, that the orientation coherence illusion is a more general byproduct of the effect of entropy on information-processing. Entropy, an information theoretic metric, quantifies the weighted average of the number of bits of information required to convey the amount of diversity or variability of mixture arrays (Shannon \& Weaver, 1949). Both human and non-human animals are sensitive to the degree of entropy in visual arrays (Wasserman \& Young, 2010; Wasserman, Young, \& Nolan, 2000; Young \& Wasserman, 1997, 2001). In the case of orientation variance, when all Gabor patches have the same orientation, the array has low 
entropy, whereas arrays with large orientation variance have high entropy since more bits of information are required to mathematically describe the orientations. DeWind et al. (2020) found that the orientation coherence effect increased with numerosity. Yet the effect of orientation variance and its interaction with numerosity may be collectively explained by the perceived entropy in the display arrays, given that when variance is held constant entropy increases with numerosity.

The second goal of the current research was to ask whether children are susceptible to the coherence illusion and how it changes with age. Prior work has shown that a variety of visual illusions increase in magnitude from childhood to adulthood, including but not limited to the Solitaire illusion (Frith \& Frit, 1972; Parrish, Agrillo, Perdue, \& Beran, 2016), the Ponzo illusion (Leibowitz \& Judisch, 1967), and the Ebbinghaus illusion (Doherty, Campbell, Tsuji, \& Phillips, 2010); whereas susceptibility to other illusions, such as the Müller-Lyer or the Delboeuf illusion (Binet, 1895; Piaget, Maire, \& Privat, 1954; Weintraub \& Cooper, 1972), decrease with age. It is possible that the role of accumulated perceptual experience (perhaps more experience in representing and manipulating quantities) is critical in the emergence and progression of the coherence illusion. Under this scenario we would expect to see a positive relationship between age and the magnitude of the coherence effect. Alternatively, given that children become better able to inhibit attention towards irrelevant non-numerical features when performing numerosity comparison tasks it is possible that the coherence effect would dissipate with development (Fuhs \& McNeil, 2013; Gilmore et al., 2013).

We conducted three experiments to examine the role of entropy on the coherence illusion using color entropy. Color is useful because it is a low-level visual attribute that can be varied independently of orientation and spacing. In Experiment 1 participants engaged in an ordinal 
numerical comparison task with maximally heterogeneous colored arrays and purely homogeneous

colored arrays. Experiment 2 tested whether color entropy parametrically affects numerosity perception. Experiment 3 tested for parametric effects of color heterogeneity again in an ordinal numerical task with 5-17-year-old participants. In the General Discussion we consider a novel resource-rational model for understanding the coherence illusion as well as the computational mechanisms underlying our visual number sense.

\section{Experiment 1}

Experiment 1 was designed to examine whether color coherence affects numerosity perception. Specifically, we tested whether a homogenously colored array is perceived as more numerous than a heterogeneously colored array.

\subsection{Participant}

Forty subjects were recruited from a large university community in exchange for course credit $\left(M_{\text {age }}=20.29\right.$ years; 26 females $)$. One participant with mean accuracy $(52 \%)$ lower than three standard deviations from the group mean was excluded. All participants had normal or corrected-to-normal visual acuity and normal color vision.

\subsection{Materials and design}

Stimuli were generated and displayed using MATLAB with Psychophysics Toolbox-3 extension on a Dell personal computer (Brainard, 1997; Pelli, 1997). The monitor was $51 \times 28.5$ $\mathrm{cm}$ with a resolution of $1,920 \times 1,080$ pixels. Participants were at an average viewing distance of $56 \mathrm{~cm}$ from the screen.

Each stimulus array was composed of 8-32 dots (each 60-pixel in diameter) on a neutral grey background. We selected 8 highly distinguishable colors by using the "Generate maximally 
perceptually-distinct colors" MATLAB function ${ }^{1}$, including the following RGB combinations: $(0$, $0,1),(0,1,0),(1,0,0),(1,0,0.72),(1,0.82,0),(0,0,0.34),(0,1,0.79)$, and $(0,0.58,1)$. The dots within each array were either of the same color (homogeneous) or filled with a full set of 8 colors (heterogeneous). Although there were only 8 colors and arrays had 8-32 dots, all 8 colors were used in all heterogeneous arrays.
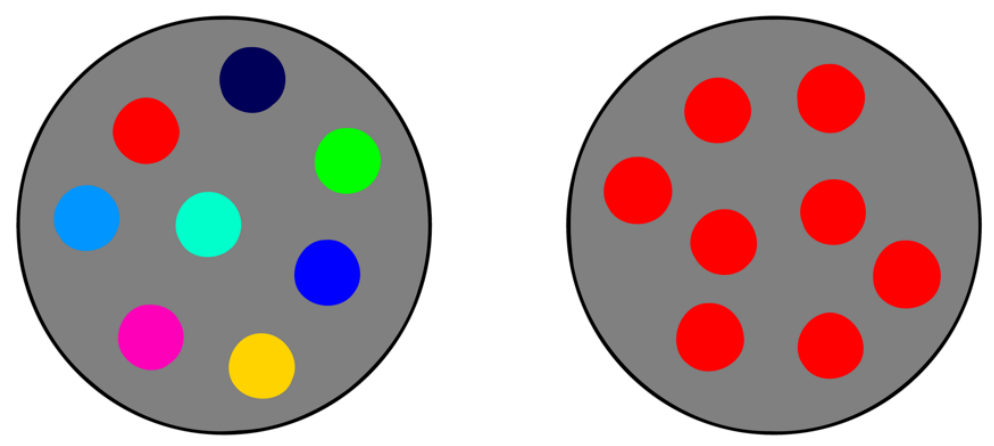

Figure 1. An illustrative example of two sample arrays in Experiment 1. Both arrays contain 8 dots of equal size. The array on the left side contains dots of 8 different colors (heterogeneous), while the array on the right side contains 8 dots of the same color (homogeneous).

There were four different trial types: 1) two homogeneously colored arrays; 2) two heterogeneously colored arrays; 3 ) one heterogeneous (right side) and one homogeneous (left side) array 4) one homogeneous (right side) and one heterogeneous (left side) array. Each type of stimuli had equal probability of appearing on each trial.

Numerical values were chosen to be approximately evenly spaced on a log scale $(8,10,11$, $13,16,19,23,27,32)$. Accordingly, the same distance between any two numerosities on the $\log$ scale corresponded to roughly the same ratio regardless of the absolute numerical values. For example, a trial with arrays of 11 and 13 circles has a similar ratio to a trial with arrays of 16 and

\footnotetext{
${ }^{1}$ This function was downloaded on https://www.mathworks.com/matlabcentral/fileexchange/29702-generatemaximally-perceptually-distinct-colors
} 
19 circles, because both pairs are spaced one unit apart on the log scale. The numerical ratio levels between the two arrays were evenly separated in a base- $2 \log$ space from 0 to 1 , yielding 5 ratio values: $1\left(=2^{0}\right), 1.19\left(=2^{0.25}\right), 1.41\left(=2^{0.5}\right), 1.68\left(=2^{0.75}\right)$, and $2\left(=2^{1}\right)$. We included an equal number of trials at each ratio level. This allowed the ratio between the two arrays to be independent of the numerical value of the arrays. Each participant completed 600 trials in total, with 120 trials at each numerical ratio and 150 trials of each trial type.

\subsection{Procedure}

Participants completed consent and demographic forms before the start of the task. Each trial started with a 500-ms fixation screen. Then two arrays were presented simultaneously for 750-ms, followed by a response cue. Participants were instructed to press the left or right arrowkey on the keyboard to indicate which array contained the greater number of circles. While participants were allowed an unlimited amount of response time, they were instructed to respond as quickly and accurately as possible. The whole experiment lasted approximately 45 minutes.

\subsection{Data analysis - generalized linear model (GLM)}

A generalized linear model (GLM) was built to quantify the effects of numerical ratio and color variance on participants' item-level binary responses. Specifically, we fit the GLM using a probit link function and a binomial error distribution with a constant term and regressors for logarithm of the numerical ratio and the difference in color variance between the two arrays.

$$
p(\text { choose right })=\Phi\left(\beta_{\text {Side }}+\beta_{\text {Num }} \log _{2}\left(r_{N u m}\right)+\beta_{\text {Col }} \text { ColorVarDiff }\right)
$$

where $p$ (choose right) is the probability of choosing the stimulus on the right-side of the screen, $\Phi$ is the cumulative distribution function of the standard normal distribution, $\beta_{\text {Side }}$ is the $\mathrm{y}$ intercept indicating subject's biased preference for choosing one side over the other regardless of the number of items or color coherence, $\beta_{N u m}$ and $\beta_{C o l}$ are the regression coefficients fit to the 
numerical ratio and color variance regressors, respectively. $r_{N u m}$ is the ratio of the number of the right array to the number of the left array, which varied from $1 / 2$ to 2 . A positive $\beta_{N u m}$ indicates that participants mostly choose the side with greater number of items, which characterizes the precision on the numerosity comparison task. ColorVarDiff is the variable indicating color variance difference, which is coded according to the following rules with three possible values: 1 if the right side was heterogeneous, and the left side was homogeneous, -1 if the left side was heterogeneous, and the right side was homogeneous, or 0 if two sides were of the equal color variance (both heterogeneous or homogeneous). Accordingly, a negative $\beta_{\mathrm{Col}}$ indicates that the less variable side is perceived to be more numerous. Each participant's binary choice data was modeled separately.

To quantify the color variance effect with respect to the numerical ratio, we then solved for $r_{N u m}$ using the mean coefficient estimates from the regression model (Equation 1) across participants. Specifically, given $\beta_{N u m}, \beta_{C o l}$, and a maximum color variance difference $(-1$ or 1 in this case), we used the following equation to find $r_{N u m}$ that would equivalently compensate for the perceived numerosity difference between the uniformly colored array and variably colored array.

$$
\beta_{\mathrm{Num}} \log _{2}\left(r_{\mathrm{Num}}\right)=\beta_{\mathrm{Col}} \text { ColorVarDiff }
$$

\subsection{Results and discussion}

Thirty-nine participants performed the task with above chance accuracy ${ }^{2}$ (Accuracy $\mathrm{M}=$ $93.3 \%, \mathrm{SD}=0.03,95 \% \mathrm{CI}[.93, .94], \mathrm{t}_{38}=107.4, \mathrm{p}<.001 ;$ One sample t-test). The regression

\footnotetext{
${ }^{2}$ Accuracy was calculated as the proportion of correctness across trials excluding those with a 1:1 ratio, where there were no correct answers. These trials were nevertheless included for regression modeling analysis because they were highly informative of the strength of the color variance effect.
} 
model (Equation 1) was fit to each participant's binary response data. We ran a one-sample t-test to determine whether the regression coefficient of each variable was significantly different from zero across participants. $\beta_{\text {Side }}$ was not significantly different from zero, indicating that participants showed no biased preference of choosing one side over the other $\left(\beta_{\text {Side }} \mathrm{M}=-0.05, \mathrm{SEM}=0.08\right.$, $\left.95 \% \mathrm{CI}[-.21, .11], \mathrm{t}_{38}=-0.60, \mathrm{p}=0.55\right)$. The mean regression coefficient of numerical ratio $\beta_{N u m}$ was significantly positive, indicating that participants' performance on numerosity discrimination was strongly modulated by the ratio between the two numbers $\left(\beta_{\text {Num }} \mathrm{M}=6.32, \mathrm{SEM}=0.22,95 \%\right.$ CI $\left.[5.88,6.77], \mathrm{t}_{38}=29.03, \mathrm{p}<.001\right)$. Consistent with our hypothesis, we found a robust effect of color variance on participants' binary response data: the mean coefficient of the color variance difference $\beta_{\text {Col }}$ was significantly below zero $\left(\beta_{\text {Col }} \mathrm{M}=-0.47, \mathrm{SEM}=0.06,95 \% \mathrm{CI}[-.59,-.35], \mathrm{t}_{38}\right.$ $=-7.79, \mathrm{p}<.001)$. This indicates that heterogeneous arrays were perceived as less numerous whereas homogeneous arrays appeared to be more numerous. The regression model (Equation 1) was also fit to the pooled data across participants to plot the psychometric curves in Figure 2 . The sigmoid curve of numerical ratio systematically shifts to the left when the right side is homogeneous indicating that homogeneity increased perceived numerosity. Overall, 35 out of 39 participants $(89.7 \%)$ exhibited a negative coefficient for color variance difference $\left(\beta_{\text {Col }}\right)$. The uniformly colored arrays were perceived as 5.0\% more numerous than the variably colored arrays (Equation 2). Correspondingly, this effect would result in 19 dots of the same color being psychologically equivalent to 20 dots of variable colors. 


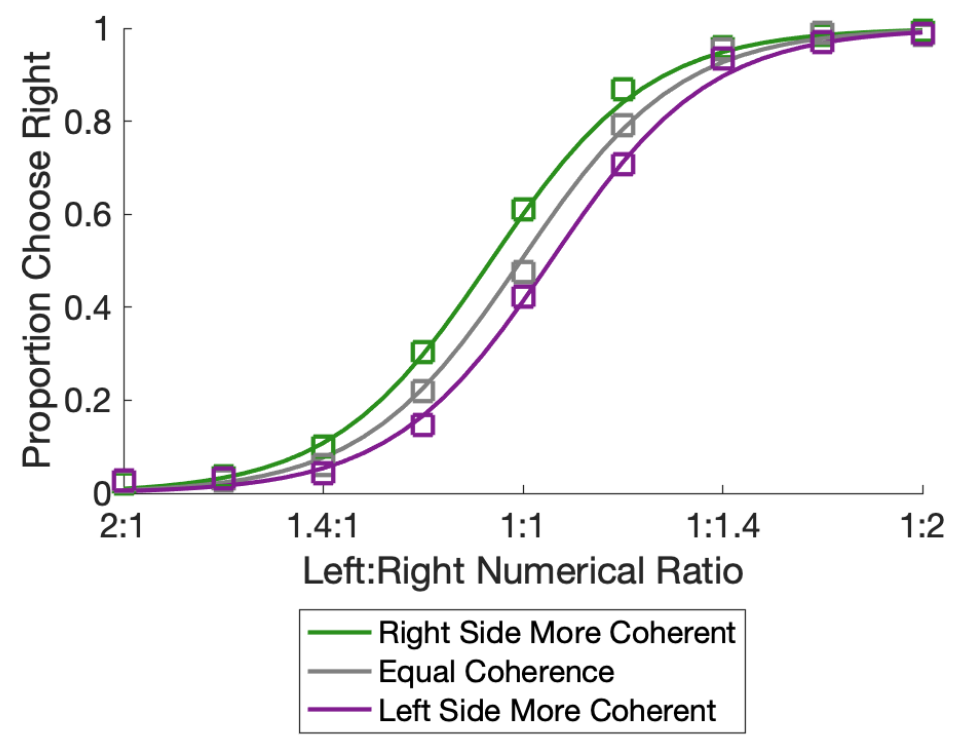

Figure 2. Homogeneously colored arrays were perceived as more numerous. The proportion of choosing the right side as a function of the left-to-right numerical ratio. The curves represent the smooth fit of the regression model in Equation 1 to the pooled data. Green line indicates more coherence (lower variance) on the right side, purple line indicates more coherence (lower variance) on the left side, grey line indicates equal coherence of both sides. The squares represent the mean proportion of choosing the right at each combination of numerical ratio levels and color variance difference conditions across participants.

To further examine whether the color entropy effect interacts with the precision of numerosity comparison, we ran an additional generalized linear mixed-effects model (GLMM). This GLMM followed a binomial error distribution with a logit link function predicting participants' item-level binary responses (left or right) from the numerical ratio, the color variance difference, and the interaction between these two fixed predictors while controlling for the random effects of participants. Consistent with our previous finding, we found significant fixed effects of both numerical ratio and color variance difference $\left(\beta_{N u m}=6.20, \mathrm{SE}=0.20, \mathrm{t}_{23396}=30.49, \mathrm{p}<.001\right.$, $95 \%$ CI $[5.80,6.60] ; \beta_{C o l}=-0.46, \mathrm{SE}=0.06, \mathrm{t}_{23396}=-7.83, \mathrm{p}<.001,95 \%$ CI $\left.[-.57,-.34]\right)$. The interaction between these two fixed effects was not significant $\left(\beta_{N u m} \times \mathrm{Col}=0.12, \mathrm{SE}=0.12, \mathrm{t}_{23396}\right.$ $=1.05, \mathrm{p}=.29)$, indicating that the color variance did not influence the precision of the numerosity 
comparison task. To interpret the absolute change in the likelihood of choosing the right side for each unit of color variance difference, we calculated the odds ratio for color variance difference by exponentiating model coefficient. Note that there were three levels of color variance difference coded as $-1,0$ and 1 . Results showed that one unit increase in color variance difference between the right-side array and the left-side array led to a $36.8 \%$ decrease in the odds of picking the right side.

\section{Experiment 2}

In Experiment 1, we found a robust effect of color uniformity on numerosity discrimination performance. Specifically, the homogeneous colored arrays were perceived to be more numerous than the heterogeneous colored arrays. Experiment 2 was designed with three goals. First, we wanted to rule out the alternative possibility that participants were simply more attracted to perfect color homogeneity. Second, we wanted to replicate the color coherence effect in an independent sample with an online platform. Third, instead of presenting only homogeneous and maximally heterogenous arrays we systematically manipulated color entropy to assess whether color entropy had a parametric effect on perceived numerosity.

\subsection{Participants}

Thirty-two adults ( $M_{\text {age }}=26.77$ years, $S D=10.86$ years, 24 females $)$ participated in an online experiment through Pavlovia in exchange for \$25. All participants reported normal or corrected-to-normal visual acuity and color vision. One participant completed 1 out of the 4 experimental sessions and three participants accidentally completed extra sessions. Nevertheless, all 32 participants were included in our data analyses.

\subsection{Materials and design}


The methods were identical to Experiment 1 except for the following deviations. While the same eight highly distinguishable colors were used, rather than including only homogeneous or heterogeneous arrays we manipulated the entropy of arrays by varying the number of color categories and the number of dots in each color category. Entropy was formally defined based on information theory to quantify the information content and was calculated as follows (Shannon \& Weaver, 1949):

$$
\text { Entropy }=-\sum_{i=1}^{n} p_{i} \log _{2} p_{i}
$$

where $p_{i}$ is the proportion of individual dots belonging to the $i$ th category of color in an array, and $n$ is the total number of color categories. Homogeneous arrays have an entropy of 0 . Maximally heterogeneous arrays were eight-dot displays with eight different colors (entropy $=-0.125 \times \log _{2}(-$ $0.125) \times 8=3.0$ ). More broadly, entropy is maximized in arrays where all colors appear equally frequently (e.g., 24 dot arrays with three of each of the eight colors). Entropy values ranged from 0 to 3 and were equal or close to one of seven values $(.0,0.5,1,1.5,2.0,2.5$, and 3.0$)$, following Experiment 2 of Young and Wasserman (2001). Accordingly, entropy differences between the left and right arrays ranged from -3 to 3 , resulting in 13 levels. The same numerical values $(8,10,11$, $13,16,19,23,27$, and 32) and the same ratio levels were used as in Exp. 1.
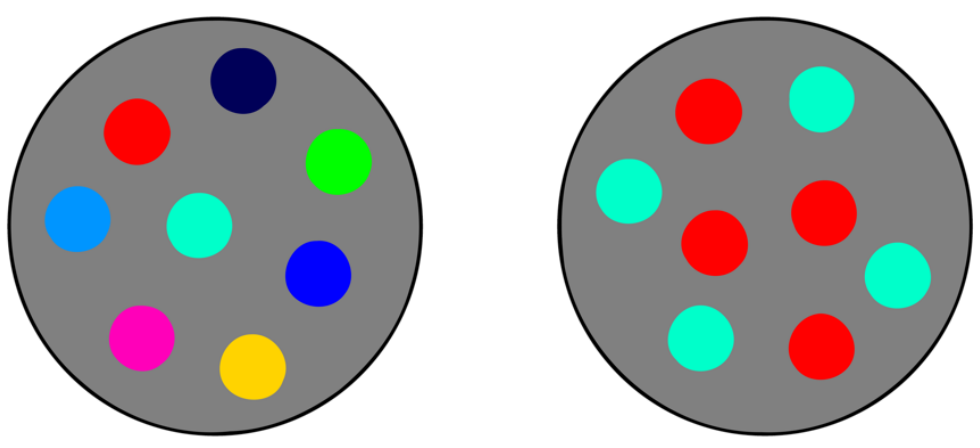
Figure 3. An illustrative example of two sample arrays for Experiment 2 and 3. Both arrays contain 8 dots of equal size. The array on the left side has 8 variably colored dots $($ entropy $=3$ ), while the array on the right side has 4 dots of each of the 2 different color categories (entropy $=$ 1).

To obtain paired displays with an even distribution of numerical ratios and entropy differences, we developed an algorithm to generate all possible colored displays, each with a unique combination of numerical values and color entropy. We then filtered these pairs to ensure that there were the same number of trials corresponding to each numerical ratio level and each difference-in-entropy level. Specifically, we included 120 paired displays at each of the 5 numerical ratio levels and approximately 46 stimuli at each of the 13 difference-in-entropy level, resulting in 600 trials in total. The task generated the paired stimuli by randomly localizing dots within the two arrays and randomly picking which colors to be included.

Because this experiment was conducted online, we were unable to control monitor sizes and screen resolutions. However, we specified the stimulus units relative to the height of the window so that the stimuli could scale naturally with the window size. This ensured that all browsers allowed the participants to see the full screen window with an appropriate pixel size of the display. Participants were given the same instructions and task as described for Experiment 1.

\subsection{Data analysis - generalized linear model (GLM)}

We fit the following generalized linear model (GLM) to participants' binary choice data (left or right) using a probit link function and a binomial error distribution with the log of the numerical ratio between the two arrays and the difference in entropy between the two arrays entered as regressors:

$$
p(\text { choose right })=\Phi\left(\beta_{\text {Side }}+\beta_{\text {Num }} \log _{2}\left(r_{\text {Num }}\right)+\beta_{\text {Col ColorEntropyDiff })}\right.
$$


The main deviations from the GLM used in Experiment 1 are that the ColorEntropyDiff is the difference in entropy between the two arrays in the paired displays, calculated as entropy of the right-side array minus the entropy of the left-side array; and $\beta_{\mathrm{Col}}$ is now the regression coefficient fit to the entropy difference regressor, which indicates the color entropy effect. Each participant's binary response data was modeled separately.

To intuitively represent the magnitude of the color entropy effect, we then solve for $r_{N u m}$ that would be sufficient to offset the perceived numerosity difference when the color entropy difference between the two arrays is maximum ( -3 or 3$)$, using the average coefficient estimates based on the regression model (Equation 3) across participants.

$$
\beta_{\text {Num }} \log _{2}\left(r_{N u m}\right)=\beta_{\text {Col }} \text { ColorEntropyDiff }
$$

\subsection{Results and discussion}

Participants performed the task with well above chance accuracy (Accuracy $M=91.3 \%$, $\mathrm{SD}=0.06,95 \% \mathrm{CI}[.89, .93], \mathrm{t}_{31}=42.3, \mathrm{p}<.001$; One sample $\mathrm{t}$-test $)$. To quantify the effect of the color coherence illusion, we fit the regression model (Equation 3) to each participant's binary response data. We next ran a one-sample t-test to examine if the coefficients of each regressor were significantly different from zero across participants. As in Experiment 1, there was no significant side bias $\left(\beta_{\text {Side }} \mathrm{M}=-0.03, \mathrm{SEM}=0.05,95 \%\right.$ CI $\left.[-.12, .07], \mathrm{t}_{31}=-0.57, \mathrm{p}=0.57\right)$. The mean $\beta_{\text {Num }}$ was significantly positive indicating a robust effect of numerical ratio on task performance: $\left(\beta_{\text {Num }} \mathrm{M}=3.06, \mathrm{SEM}=0.13,95 \% \mathrm{CI}[2.80,3.32], \mathrm{t}_{31}=23.96, \mathrm{p}<.001\right)$. Consistent with our prediction, we also found a strong effect of color entropy $\left(\beta_{\text {Col }} \mathrm{M}=-0.09, \mathrm{SEM}=0.02\right.$, $95 \%$ CI $\left.[-.12,-.05], \mathrm{t}_{31}=-5.59, \mathrm{p}<.001\right)$. The $\beta_{\text {Col }}$ was significantly below zero, indicating that participants were more likely to perceive the right side as more numerous when the array on that 
side had lower entropy (more coherence). Twenty-six out of 32 participants $(81.3 \%)$ exhibited a negative $\beta_{C o l}$ coefficient. On average, the most coherent array (entropy $=0$ ) was perceived to be $6.0 \%$ more numerous than the most entropic array (entropy $=3)$. More intuitively, an array with an entropy of 3.0 and 17 dots would seem to be as equally numerous to an array with an entropy of 0 and 16 dots.

As can be seen in Figure 4 entropy parametrically influenced perceived numerosity. Specifically, the sigmoid curve of numerical ratio systematically shifts to the right when the right side is more entropic indicating that the right side was chosen less when the array on that side had higher entropy (less coherence).

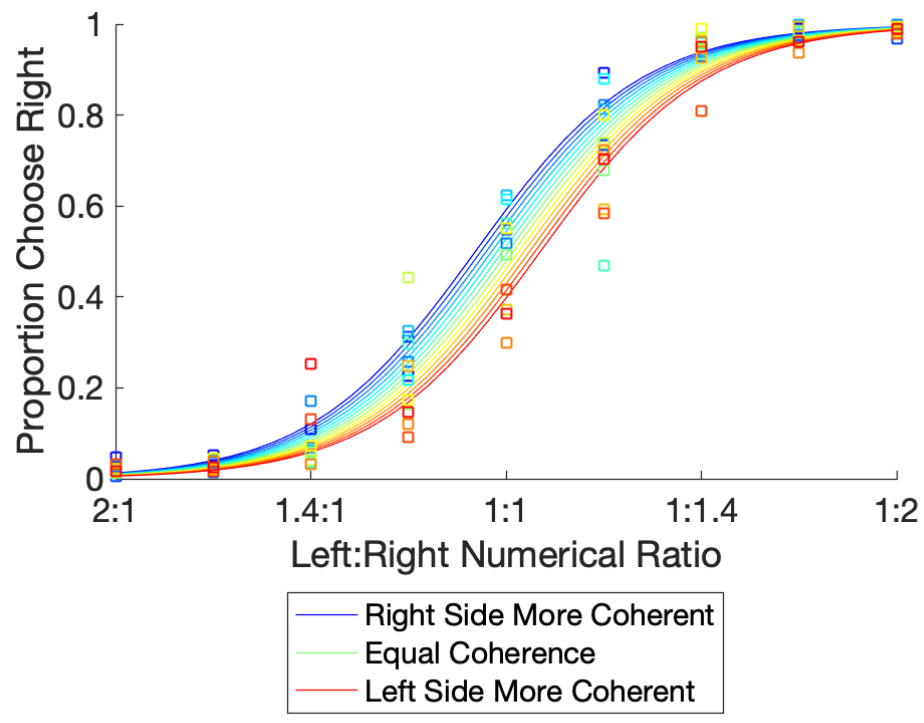

Figure 4. Lower-entropy arrays were perceived as more numerous. The curves of the proportion of choosing the right as a function of the left-to-right numerical ratio systematically shifted along the spectrum of the color entropy difference. The 13 lines represent the smooth fit of the regression model (Equation 3) to the pooled data, with each line corresponding to one difference-in-entropy level. Blue indicates more coherence (lower entropy) on the right side, red indicates more coherence (lower entropy) on the left side. The squares represent the proportion of choosing the right at each combination of numerical ratio level and difference-in-entropy level across participants. 
A GLMM to control for random effect of participants revealed a similar pattern as Experiment 1. The numerical ratio and entropy difference were both significant predictors of the item-level responses of choosing the right side $\left(\beta_{N u m}=5.47, \mathrm{SE}=0.24, \mathrm{t}_{19346}=22.72, \mathrm{p}<.001\right.$, 95\% CI $[5.00,5.95] ; \beta_{\text {Col }}=-0.15, \mathrm{SE}=0.03, \mathrm{t}_{19346}=-5.59, \mathrm{p}<.001,95 \%$ CI $\left.[-.20,-.10]\right)$. The interaction between these two fixed effects was not significant $\left(\beta_{N u m} \times \mathrm{Col}=-0.04, \mathrm{SE}=0.04, \mathrm{t}_{19346}\right.$ $=-1.00, \mathrm{p}=.32$ ), which indicates that color coherence did not influence the precision of the numerosity comparison task. Exponentiated model coefficients for fixed effects revealed that a single incremental change in color entropy difference between the right-side array and the left-side array led to a $14.0 \%$ decrease in the odds of picking the right side.

\section{Experiment 3}

Experiment 2 demonstrated that color entropy parametrically affects numerosity perception. The objective of Experiment 3 was to examine the developmental trajectory of the coherence illusion in a sample of children aged 5 to 17 . We used the same task and online platform used in Experiment 2 to allow direct comparison with data collected from adults.

\subsection{Methods}

Seventy-six children completed the online experiment. Participants were recruited through Pavlovia and were compensated with $\$ 25$. Forty-four participants were female, and the mean age was $13.04(\operatorname{Min}=5.39, \operatorname{Max}=18.00, S D=3.99)$. All participants had normal or corrected-tonormal visual acuity and normal color vision. Four participants accidentally completed extra sessions and were nevertheless included. An additional eight children were recruited but were excluded from the final sample because they did not complete the 4 experimental blocks. The 
stimulus design, task procedure and regression modeling analyses were identical to those of Experiment 2.

\subsection{Results and discussion}

All children performed the task with above chance accuracy (Accuracy $\mathrm{M}=86.9 \%, \mathrm{SD}=$ $0.08,95 \% \mathrm{CI}[.85, .89], \mathrm{t}_{75}=38.4, \mathrm{p}<.001$; One-sample t-test). Each participant's binary response data was modeled separately using Equation 3. Similar to adults, children showed no side bias $\left(\beta_{\text {Side }} \mathrm{M}=-0.02, \mathrm{SEM}=0.02,95 \% \mathrm{CI}[-.05, .02], \mathrm{t}_{75}=-0.87, \mathrm{p}=0.39\right)$. Like adults, children also showed both a strong effect of numerical ratio with the mean $\beta_{N u m}$ being significantly positive $\left(\beta_{\text {Num }} \mathrm{M}=2.40, \mathrm{SEM}=0.11,95 \%\right.$ CI $\left.[2.18,2.61], \mathrm{t}_{75}=22.56, \mathrm{p}<.001\right)$ and a significant effect of color entropy difference $\left(\beta_{\text {Col }} \mathrm{M}=-0.07, \mathrm{SEM}=0.01,95 \% \mathrm{CI}[-.09,-.05], \mathrm{t}_{75}=-7.80, \mathrm{p}<.001\right)$. Equation 4 revealed that the most coherent array (entropy $=0$ ) was perceived to be $6.0 \%$ more numerous than the most entropic array (entropy $=3$ ).

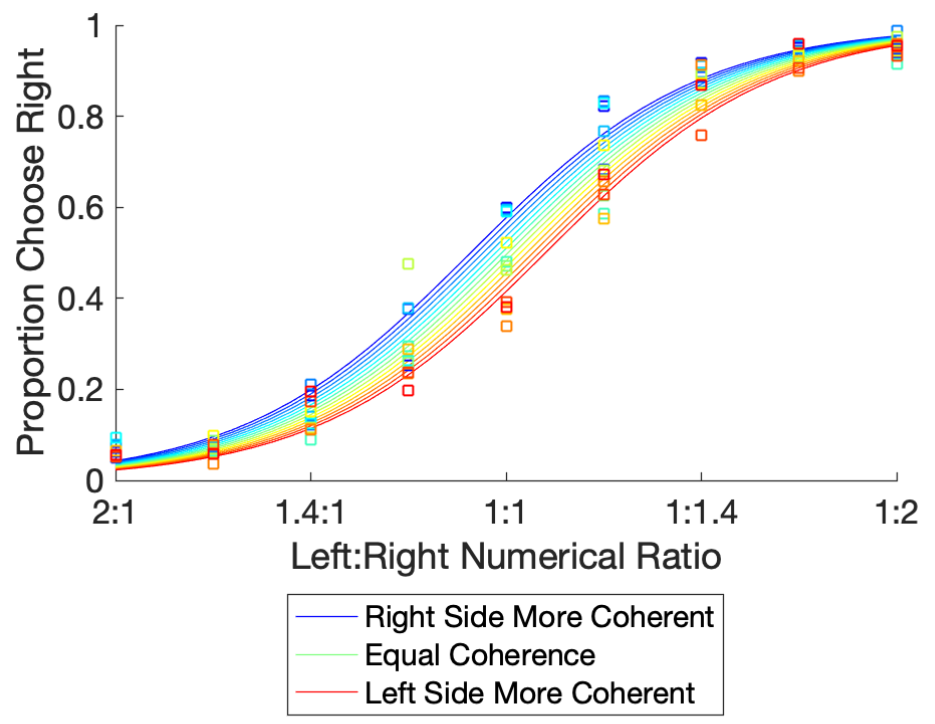

Figure 5. Replication of the color entropy effect in a large sample of children. The conventions are the same as in Figure 4. 
As can be seen in Figure 5 entropy parametrically influenced perceived numerosity for children. Specifically, the sigmoid curve of numerical ratio systematically shifted to the right when the right side was more entropic indicating that the right side was chosen less when the array on that side had higher entropy. A GLMM with individuals treated as a random effect revealed significant fixed-effects of numerical ratio $\left(\beta_{N u m}=4.23, \mathrm{SE}=0.20, \mathrm{Z}=21.69, \mathrm{p}<.001,95 \% \mathrm{CI}\right.$ $[3.84,4.61])$ and color entropy difference $\left(\beta_{\mathrm{Col}}=-0.12, \mathrm{SE}=0.02, \mathrm{Z}=-8.12, \mathrm{p}<.001,95 \% \mathrm{CI}\right.$ $[-.15,-.09])$. One unit difference in color entropy between the left and right side led to a $11.7 \%$ change in the odds of indicating that the right side was more numerous. Similar to adults, there was no interaction between the fixed effect of numerical ratio and that of color entropy difference $\left(\beta_{N u m \times C o l}=-0.03, \mathrm{SE}=0.02, \mathrm{Z}=-1.59, \mathrm{p}=.11\right)$, indicating that the precision on numerosity comparison was not influenced by the color entropy effect.

To examine the developmental trajectory of the color entropy effect on numerical processing we analyzed the data from Experiment 3 together with data from adults aged 18-30 tested in Experiment $2^{3}$. As shown in Figure 6a, there was a strong correlation between age and the regression coefficient of numerical ratio $\left(\mathrm{r}=.60, \mathrm{t}_{99}=7.39,95 \% \mathrm{CI}[.45, .71], \mathrm{p}<.001\right)$, replicating many previous findings that nonverbal numerical precision continues to improve throughout the school-age years (Halberda \& Feigenson, 2008; Halberda, Ly, Wilmer, Naiman, \& Germine, 2012). Figure 6b shows that the coefficient of color entropy difference was negatively correlated with age $\left(r=-.24, t_{99}=-2.49,95 \%\right.$ CI $\left.[-.42,-.05], p=.01\right)$, suggesting that the magnitude of the color entropy effect increases into adulthood.

\footnotetext{
${ }^{3}$ Seven adults aged between 30 and 63 years were not included into the correlation analysis, since the limited sample size in this wide age range would obscure the developmental trajectory.
} 

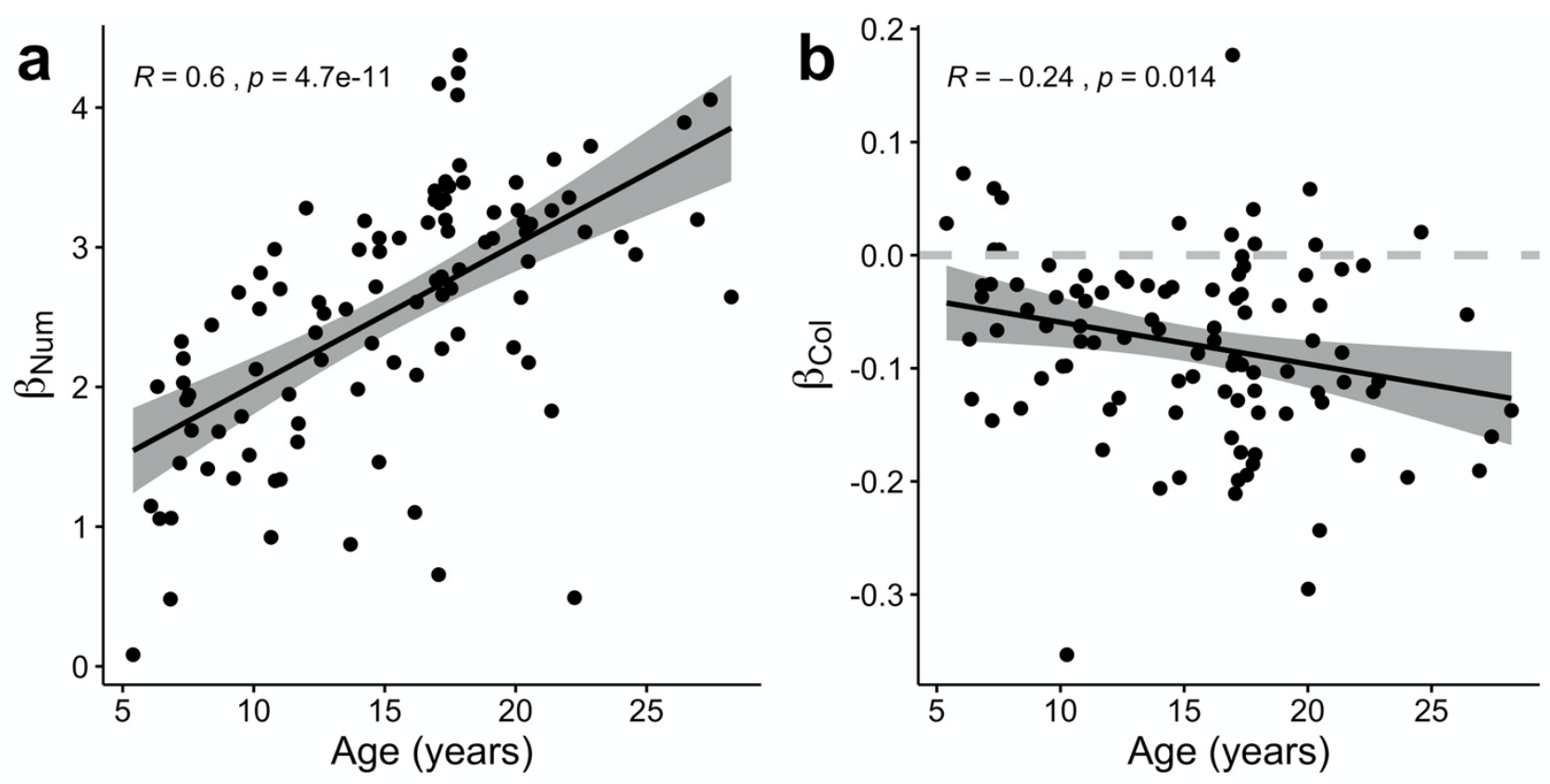

Figure 6. Scatter plots of zero-order correlations between age and each of the regression coefficients. (a) The coefficient of numerical ratio was positively correlated with age. The grey band region denotes $95 \%$ confidence intervals. (b) The coefficient of color entropy difference was negatively correlated with age, indicating that the magnitude of the color coherence illusion increases over development. The grey dashed line represents coefficient value of zero.

\section{General Discussion}

Illusions that emerge as a byproduct of systematic errors in processing numerical information serve as a powerful window into the mechanisms of numerosity perception (e.g., Eagleman, 2001; Parrish et al., 2016). To test the generality of our previously reported coherence illusion and to gain further insight into how numerosity is extracted from visual scenes we tested the influence of color entropy on numerosity perception. In Experiment 1 we showed that homogeneously colored arrays were perceived as more numerous than heterogeneously colored arrays. In Experiment 2 we parametrically varied color entropy and found systematic effects on numerosity perception. In Experiment 3, we replicated the parametric effect of color entropy on perceived numerosity in a large sample of children aged 5 to 17 and found that the susceptibility to the coherence illusion was present even in our youngest participants but also increased with age. 
Our investigation replicates the coherence illusion first described by DeWind et al. (2020) and demonstrates that this illusion is not specific to orientation but instead that the entropy of visual arrays more generally influences numerical perception.

An intriguing possibility is that display variability, including but not limited to variance in orientation or color, is directly encoded. When adapted to arrays with a given orientation variance people show adaptation aftereffects on subsequent orientation variance judgments (Norman et al., 2015). Remarkably, adaptation to variance can generalize across visual domains. When adapted to arrays with a given color variance, judgements of orientation variance were systematically changed (Maule \& Franklin, 2020). It is important to note that although both entropy and variance represent the degree of randomness or diversity of a visual display, entropy is a more precise psychophysical metric that quantifies graded change in display variability (Shannon \& Weaver, 1949). DeWind et al (2020) reported an interaction whereby the magnitude of the orientation variance effect on numerosity judgement increased with the magnitude of the numerosity (DeWind et al., 2020). This interaction likely reflects the fact that orientation entropy increases with numerosity even when variance is held constant. For example, while an 8-item array with 8 distinct radians has the same variance as a 32-item array with 32 distinct radians, the 32-item array has higher entropy. Future research should disentangle the psychological significance of variance and entropy.

While this series of studies was not designed to address the debate over whether number is directly or indirectly perceived, we note that our findings are problematic for indirect models. We found that increasing the degree of entropy, without changing non-numerical magnitudes, deflated perceived numerosity. If number were indirectly derived from other magnitude representations perceived numerosity should not vary with entropy given that all non-numerical magnitudes were kept constant across levels of entropy. It is, however, conceivable that entropy influences 
perceived numerousness by changing the subjective experience of other magnitudes. For example, perhaps high entropy arrays are perceived as having lower inter-element spacing and this results in lower perceived numerousness via the occupancy model (Allik \& Tuulmets, 1991).

Although the comparison task we used in the present studies cannot resolve whether coherence yields increased perceived numerosity or entropy yields decreased perceived numerosity, we currently favor the latter interpretation. In the previous report of the orientation coherence effect participants in one of the four experiments estimated array numerosity by typing a number into a computer keypad (see Figure 5 DeWind et al., 2020). Consistent with many prior studies, on average participants underestimated numerosity (e.g., Izard \& Dehaene, 2008). However, in that study arrays with higher orientation variance were underestimated to a greater degree than arrays with low variance. A recently proposed Bayesian resource-rational model provides a potential framework for understanding why low entropy arrays would be perceived as more numerous than high entropy arrays (Cheyette \& Piantadosi, 2020). The model posits that our perceptual system is optimized for efficiency and that information about numerosity is extracted from the retina at a finite rate. Any manipulation that reduces the rate of information transfer leads to underestimation because with less information about the stimulus observers are thought to rely more heavily on priors. Priors for numerosity are assumed to over-represent small numerosities because of their prevalence in the world. For example, when Cheyette and Piantadosi (2020) experimentally increased display contrast this led to a reduction in underestimation and thus more veridical estimates. Entropy or increased variance may reduce the rate of numerical information transfer (r) and consequently lead to underestimation of numerosity.

Finally, we explored whether there is a shift in susceptibility to the coherence illusion over development. One hypothesis is that the coherence illusion would be attenuated with age-related 
improvements in executive functioning skills that allow filtering of irrelevant cues. As children age they are better able to ignore irrelevant non-numerical features such as surface area and attend to number in non-symbolic numerical comparison tasks and so they may also be better at ignoring array entropy (Fuhs \& McNeil, 2013; Gilmore et al., 2013). In contrast to this hypothesis, we found that rather than decreasing with continual improvements in ANS acuity and executive functioning skills the coherence illusion increases in magnitude into adulthood.

The positive correlation between age and the strength of the coherence illusion raises a number of questions. One possibility is that perceptual experience over development, particularly experience in making quantitative judgements, increases the strength of the coherence illusion. Indeed, humans implicitly learn and extract many different statistical regularities of the environment, and this knowledge can bias perception through modulating information acquisition and interpretation (Peters, Balzer, \& Shams, 2015; Seriès \& Seitz, 2013; Summerfield \& Egner, 2009). If there is a statistical association in natural scenes whereby homogeneous arrays are generally more numerous this may be learned over development and may influence our numerical judgements in a manner that favored susceptibility to the coherence illusion. A second possibility in line with resource-rational model, is that the numerical encoding system may become more efficient at taking advantage of Bayesian priors with age. Specifically, when the information transfer rate is reduced by display entropy adults might rely more heavily on the need probability of number than children. To test this possibility, it would be important to know whether entropy leads to a greater degree of underestimation in adults compared with children using an estimation rather than a comparison task. Yet a third possibility is that the weaker coherence effect in younger participants reflects lower acuity in perceived entropy. If young children have less precise representations of entropy, then entropy may affect numerical judgments less. Future work could 
test entropy judgments to assess whether developmental improvements in entropy perception are associated with increased magnitude of the coherence effect or whether individual differences in entropy judgments are correlated with differences in the magnitude of the coherence effect.

Another interesting question is when in the neural processing stage entropy influences numerosity perception. Recent research indicates that numerosity is encoded extremely early in the visual stream, independently of numerical coding in higher-order areas such as the intraparietal sulcus (DeWind, Park, Woldorff, \& Brannon, 2019; Lucero et al., 2020; Park, DeWind, Woldorff, \& Brannon, 2016). It is therefore possible that entropy interacts with low-level visual cortex signals for numerosity, perhaps even before later attentive stages at which items in a collection can be consciously individuated. Determining the visual processing stage at which a robust entropy effect arises should ultimately aid in a greater understanding of the mechanisms underlying our visual number sense.

\section{Conclusions}

Across three experiments, we found that coherent colored arrays were perceived as more numerous than entropic colored arrays. Collectively, our findings and those of DeWind et al. (2020) indicate that the coherence illusion on numerosity perception is not specific to a particular visual property but instead that the entropy of visual arrays more generally affects numerical processing. Moreover, the strength of the coherence illusion increases into adulthood, thereby raising intriguing questions as to how perceptual experiences influence the emergence and progression of this numerosity illusion. Entropy, which makes information a measurable physical quantity, should serve as a powerful tool to test models of efficient numerical encoding system and even a broader 
range of other fundamental cognitive computational processes under an information-theoretic perspective. 


\section{References}

Adriano, A., Girelli, L., \& Rinaldi, L. (2021). Non-symbolic numerosity encoding escapes spatial frequency equalization. Psychological Research, 1-14.

Adriano, A., Rinaldi, L., \& Girelli, L. (2021). Visual illusions as a tool to hijack numerical perception: Disentangling non-symbolic number from its continuous visual properties. Journal of Experimental Psychology: Human Perception \& Performance.

Allik, J., \& Tuulmets, T. (1991). Occupancy model of perceived numerosity. Perception \& Psychophysics, 49(4), 303-314.

Binet, A. (1895). La mesure des illusions visuelles chez les enfants. Revue Philosophique de La France et de l'Étranger, 40, 11-25.

Brainard, D. H. (1997). The psychophysics toolbox. Spatial Vision, 10(4), 433-436.

Brannon, E. M., \& Terrace, H. S. (1998). Ordering of the numerosities 1 to 9 by monkeys. Science, 282(5389), 746-749.

Cantlon, J. F., \& Brannon, E. M. (2006). Shared system for ordering small and large numbers in monkeys and humans. Psychological Science, 17(5), 401-406.

Cantlon, J. F., Cordes, S., Libertus, M. E., \& Brannon, E. M. (2009). Comment on “ Log or Linear? Distinct. Science, 323(January), 38b.

Cheyette, S. J., \& Piantadosi, S. T. (2020). A unified account of numerosity perception. Nature Human Behaviour, 1-8.

Dakin, S. C. (1999). Orientation variance as a quantifier of structure in texture. Spatial Vision, $12(1), 1-30$.

Dakin, S. C., Tibber, M. S., Greenwood, J. A., \& Morgan, M. J. (2011). A common visual metric for approximate number and density. Proceedings of the National Academy of Sciences, 
108(49), 19552-19557.

Dehaene, S., \& Changeux, J. P. (1993). Development of elementary numerical abilities: A neuronal model. Journal of cognitive neuroscience, 5(4), 390-407.

Dehaene, S. (1997). The number sense: How the mind creates mathematics. New York: Oxford University Press.

DeWind, N. K., Adams, G. K., Platt, M. L., \& Brannon, E. M. (2015). Modeling the approximate number system to quantify the contribution of visual stimulus features. Cognition, 142, 247-265. https://doi.org/10.1016/j.cognition.2015.05.016

DeWind, N. K., Bonner, M. F., \& Brannon, E. M. (2020). Similarly oriented objects appear more numerous. Journal of Vision, 20(4), 4.

DeWind, N. K., Park, J., Woldorff, M. G., \& Brannon, E. M. (2019). Numerical encoding in early visual cortex. Cortex, 114, 76-89.

Doherty, M. J., Campbell, N. M., Tsuji, H., \& Phillips, W. A. (2010). The Ebbinghaus illusion deceives adults but not young children. Developmental Science, 13(5), 714-721. https://doi.org/10.1111/j.1467-7687.2009.00931.x

Eagleman, D. M. (2001). Visual illusions and neurobiology. Nature Reviews Neuroscience, 2(12), 920-926.

Feigenson, L., Dehaene, S., \& Spelke, E. (2004). Core systems of number. Trends in Cognitive Sciences, 8(7), 307-314.

Franconeri, S. L., Bemis, D. K., \& Alvarez, G. A. (2009). Number estimation relies on a set of segmented objects. Cognition, 113(1), 1-13.

Frith, C. D., \& Frit, U. (1972). The solitaire illusion: An illusion of numerosity. Perception \& Psychophysics, 11(6), 409-410. 
Fuhs, M. W., \& McNeil, N. M. (2013). ANS acuity and mathematics ability in preschoolers from low-income homes: Contributions of inhibitory control. Developmental Science, 16(1), $136-148$.

Gebuis, T., \& Reynvoet, B. (2012). The interplay between nonsymbolic number and its continuous visual properties. Journal of Experimental Psychology: General, 141(4), 642.

Gilmore, C., Attridge, N., Clayton, S., Cragg, L., Johnson, S., Marlow, N., ... Inglis, M. (2013). Individual differences in inhibitory control, not non-verbal number acuity, correlate with mathematics achievement. PloS One, 8(6), e67374.

Ginsburg, N. (1976). Effect of item arrangement on perceived numerosity: Randomness vs regularity. Perceptual and Motor Skills, 43(2), 663-668.

Halberda, J., \& Feigenson, L. (2008). Developmental Change in the Acuity of the "Number Sense": The Approximate Number System in 3-, 4-, 5-, and 6-Year-Olds and Adults. Developmental Psychology, 44(5), 1457-1465. https://doi.org/10.1037/a0012682

Halberda, J., Ly, R., Wilmer, J. B., Naiman, D. Q., \& Germine, L. (2012). Number sense across the lifespan as revealed by a massive Internet-based sample. Proceedings of the National Academy of Sciences, 109(28), 11116-11120.

He, L., Zhang, J., Zhou, T., \& Chen, L. (2009). Connectedness affects dot numerosity judgment: Implications for configural processing. Psychonomic Bulletin \& Review, 16(3), 509-517.

Izard, V., \& Dehaene, S. (2008). Calibrating the mental number line. Cognition, 106(3), 12211247. https://doi.org/10.1016/j.cognition.2007.06.004

Leibovich, T., Katzin, N., Harel, M., \& Henik, A. (2017). From "sense of number" to "sense of magnitude": The role of continuous magnitudes in numerical cognition. Behavioral and Brain Sciences, 40. https://doi.org/10.1017/S0140525X16000960 
Leibowitz, H. W., \& Judisch, J. M. (1967). The relation between age and the magnitude of the Ponzo illusion. The American Journal of Psychology, 80(1), 105-109.

Lucero, C., Brookshire, G., Sava-Segal, C., Bottini, R., Goldin-Meadow, S., Vogel, E. K., \& Casasanto, D. (2020). Unconscious Number Discrimination in the Human Visual System. Cerebral Cortex.

Maule, J., \& Franklin, A. (2020). Adaptation to variance generalizes across visual domains. Journal of Experimental Psychology: General, 149(4), 662.

Morgan, M., Chubb, C., \& Solomon, J. A. (2008). A 'dipper'function for texture discrimination based on orientation variance. Journal of Vision, 8(11), 9.

Morgan, M. J., Raphael, S., Tibber, M. S., \& Dakin, S. C. (2014). A texture-processing model of the 'visual sense of number.' Proceedings of the Royal Society B: Biological Sciences, $281(1790), 20141137$.

Norman, L. J., Heywood, C. A., \& Kentridge, R. W. (2015). Direct encoding of orientation variance in the visual system. Journal of Vision, 15(4), 3.

Park, J., DeWind, N. K., Woldorff, M. G., \& Brannon, E. M. (2016). Rapid and direct encoding of numerosity in the visual stream. Cerebral Cortex, 26(2), 748-763.

Parrish, A. E., Agrillo, C., Perdue, B. M., \& Beran, M. J. (2016). The elusive illusion: Do children (Homo sapiens) and capuchin monkeys (Cebus apella) see the Solitaire illusion? Journal of Experimental Child Psychology, 142, 83-95.

Pelli, D. G. (1997). The VideoToolbox software for visual psychophysics: Transforming numbers into movies. Spatial Vision, 10(4), 437-442.

Peters, M. A. K., Balzer, J., \& Shams, L. (2015). Smaller= denser, and the brain knows it: Natural statistics of object density shape weight expectations. PloS One, 10(3), e0119794. 
Piaget, J., Maire, F., \& Privat, F. (1954). The Resistance of Good Forms in the Muller-Lyer Illusion. Archives de Psychologie, 34(135), 155-201.

Scarf, D., Hayne, H., \& Colombo, M. (2011). Pigeons on par with primates in numerical competence. Science, 334(6063), 1664.

Seriès, P., \& Seitz, A. (2013). Learning what to expect (in visual perception). Frontiers in Human Neuroscience, 7, 668.

Shannon, C. E., \& Weaver, W. (1949). A mathematical model of communication. Urbana, IL: University of Illinois Press, 11.

Starr, A., DeWind, N. K., \& Brannon, E. M. (2017). The contributions of numerical acuity and non-numerical stimulus features to the development of the number sense and symbolic math achievement. Cognition, 168, 222-233.

Summerfield, C., \& Egner, T. (2009). Expectation (and attention) in visual cognition. Trends in Cognitive Sciences, 13(9), 403-409.

Wasserman, E. A., \& Young, M. E. (2010). Same-different discrimination: The keel and backbone of thought and reasoning. Journal of Experimental Psychology: Animal Behavior Processes, 36(1), 3.

Wasserman, E. A., Young, M. E., \& Nolan, B. C. (2000). Display variability and spatial organization as contributors to the pigeon's discrimination of complex visual stimuli. Journal of Experimental Psychology: Animal Behavior Processes, 26(2), 133.

Weintraub, D. J., \& Cooper, L. A. (1972). Coming of age with the Delboeuf illusion: Brightness contrast, cognition, and perceptual development. Developmental Psychology, 6(2), 187.

Xu, F., Spelke, E. S., \& Goddard, S. (2005). Number sense in human infants. Developmental Science, 8(1), 88-101. 
Young, M. E., \& Wasserman, E. A. (1997). Entropy detection by pigeons: Response to mixed visual displays after same-different discrimination training. Journal of Experimental Psychology: Animal Behavior Processes, 23(2), 157.

Young, M. E., \& Wasserman, E. A. (2001). Entropy and variability discrimination. Journal of Experimental Psychology: Learning, Memory, and Cognition, 27(1), 278. 